\title{
Subintimal Double-Barrel Restenting of an Occluded Primary Stented Superficial Femoral Artery
}

\author{
Dirk Duterloo, Paul N.M. Lohle, Leo E.H. Lampmann \\ Department of Radiology, St. Elisabeth Hospital Tilburg, Hilvarenbeekseweg 60, 5022GC Tilburg, The Netherlands
}

\begin{abstract}
In-stent re-stenosis is a frequent complication of endovascular stents, especially in the superficial femoral artery (SFA). Endovascular re-intervention of in- or peri-stent occlusive disease consists of recanilization through the occluded stent. In our case report, we describe the endovascular treatment of a previously placed stent in the SFA. We unintentionally passed the affected stent subintimally, in a double barrel fashion next to the first stent. The procedure was without any complications and with a successfull angiographic result. At one year follow-up the patient still has no complaints and the stent is still patent.
\end{abstract}

Key words: Interventional radiology—Superficial femoral artery-Endovascular stenting-Reocclusion-PIER

In-stent restenosis is a frequent complication, especially in the superficial femoral artery (SFA). If in-stent restenosis occurs there are several therapeutic options available such as conservative therapy and endovascular or surgical intervention. Endovascular reintervention of in- or peri-stent occlusive disease is one of the less invasive options. Endovascular therapy means passage through the occluded stent (recanalization) by additional means and redilatation or restenting of the lesion. In some cases passage of the occluded stent is not possible.

In this case report we describe the unsuccessful attempt to recanalize an occlusion in a stent in the SFA followed by the successful bypass of the stent using a subintimal technique. The concept of utilizing this secondary stent was to create enough radial force to keep open the neo-lumen next to the occluded stent. Although the per-interventional extraluminal recanalization (PIER) technique is usually performed for longer lesions of the SFA, in this case a shorter segment was successfully managed $[1,2]$.

An internet search for articles describing equivalent cases revealed no results. No publications on subintimal passage of a reoccluded stent and double-barrel stenting were found in the literature.

Correspondence to: Dirk Duterloo; email: Duterke1975@hotmail.com

\section{Case Report}

A 75-year-old man underwent several percutaneous transluminal angioplasty (PTA) procedures in both upper leg vessels as well as stenting of a right SFA lesion in the adductor canal after an insufficient PTA result in an other facility (Figs. 1 and 2).

The patient presented in 2004 with progressive complaints of the right leg. The patient's maximum walking distance was $800 \mathrm{~m}$ but he smoked 5-10 cigarettes a day. As consistent medication the patient was on antihypertensive drugs, $40 \mathrm{mg}$ simvastatin (10 mg Zocor, MSD) as well as $100 \mathrm{mg}$ carbasalaatcalcium (Ascal Cardio, Viatris). There was no cardiopulmonary comorbidity.

Right pedal pulses were absent with a normal pulse at the right groin. Blood pressure was $160 / 95 \mathrm{mmHg}$. The maximum walking distance was $160 \mathrm{~m}$ with a drop in the ankle/brachial index (ABI) after excercise of 0.62 to 0.23 on the right side and 0.98 to 0.55 on the left side. In conclusion, this patient had peripheral arterial occlusive disease $(\mathrm{PaOD})$ staged as Fontaine class $2 \mathrm{a}$.

A color Doppler ultrasound (CCDS) examination of the right femoropopliteal arteries revealed in- and peri-stent restenosis with a peak systolic volume (PSV) ratio of 5.7 and distal in-stent occlusion noted over $4 \mathrm{~cm}$. At that time the patient could not be persuaded to undergo any intervention and was managed conservatively.

In March 2005 the patient presented again with unchanged symptoms. On physical examination the walking distance was now $240 \mathrm{~m}$ with an ABI drop after excercise of 0.44 to 0.22 on the right side and 0.91 to 0.52 on the left side. The patient and his attending physician discussed endovascular treatment such as recanalization of the affected vessel with PTA and/or additional stenting as a proper next option for his PaOD Fontaine class $2 \mathrm{a}$ lifestyle-limiting symptoms.

In April 2005, an endovascular percutaneous intervention of the right SFA was performed at the Department of Radiology in our hospital. Because the patient was overweight an antegrade puncture was not a good option and the right SFA was approached by a crossover technique from the contralateral side. The right leg vessels were visualized angiographically demonstrating a stenosis of approximately $70 \%$ at the origin on the right SFA. More distally in the SFA an occlusion was noticed reaching from 4 $\mathrm{cm}$ proximal of the occluded stent to the upper border of the patella (The total length of this lesion was approximately $11 \mathrm{~cm}$.) The 5 Fr Brite Tip sheath (Cordis, New York, USA) in the right femoral artery was changed for a long 6 Fr Balkin Up \& Over flexorsheath (Cook, Bloomington, IN, USA). The sheath tip was placed in the left external iliac artery, just above the hip joint. First a PTA was performed at the origin of the SFA with a 6.0/ $40.0 \mathrm{~mm}$ balloon (Pheron, Biotronik, Switzerland) after an intravenous (IV) injection of 2,500 IU heparin, with a satisfactory angiographic result. 

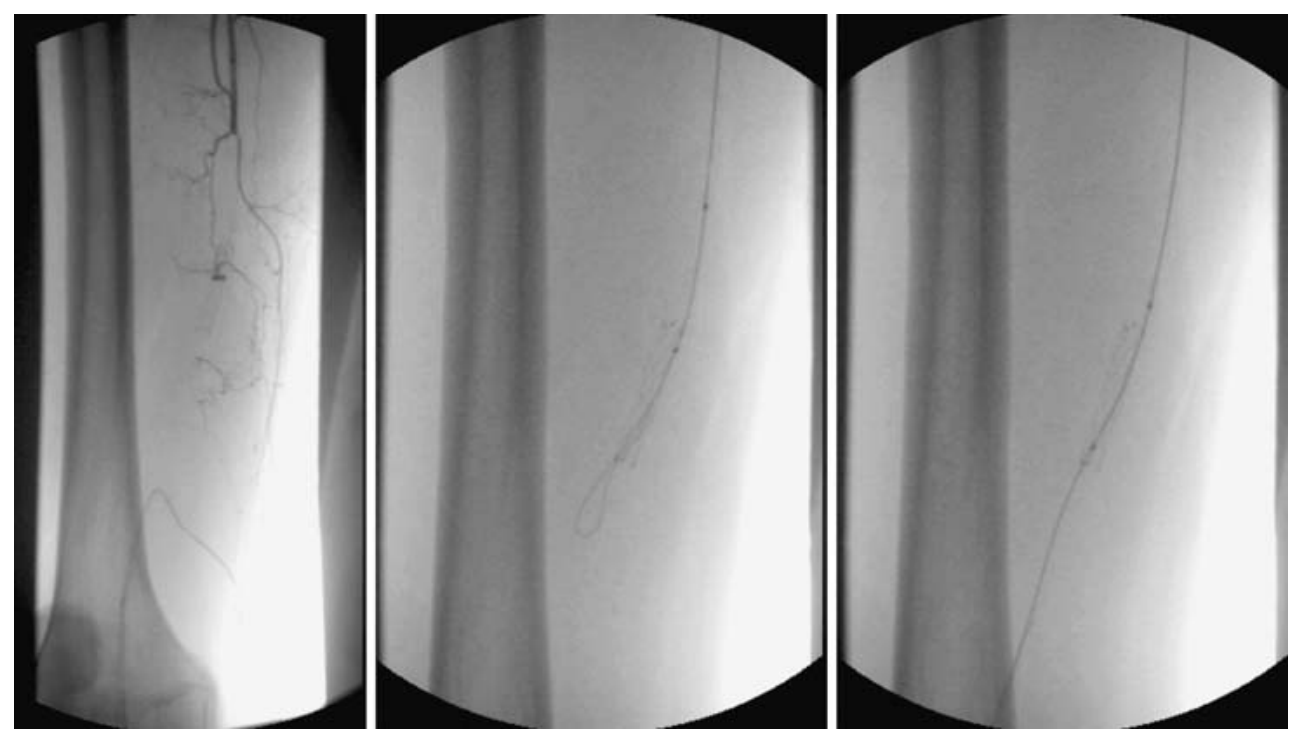

Fig. 1. See text.

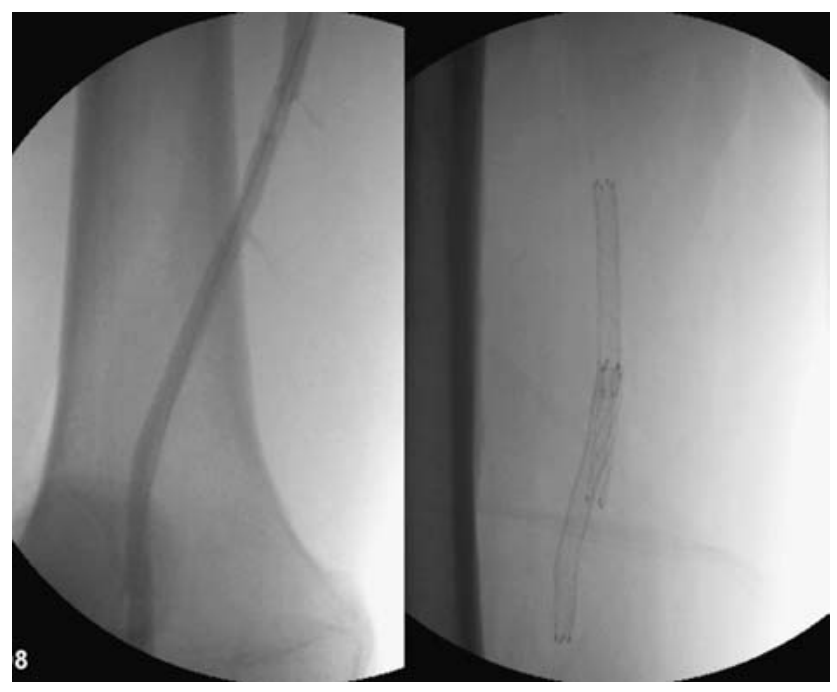

On his planned check-up at the department of surgery in May 2005 the patient reported himself very satisfied with the result. The pain sensations had disappeared and there were no more limitations to his walking distance. In August 2005 his ABI on the right side was 0.85 with a drop, after exercise, to 0.25 . On the left side his ABI was 0.88 with a drop, after exercise, to 0.62 . On color Doppler ultrasound investigation a patent secondary stent segment and no new stenotic lesions were observed and a normal flow pattern was described.

\section{Discussion}

Peri- and in-stent restenosis is a common and well-known complication in the SFA region. There are several therapeutic options to solve this problem including endovascular treatment and bypass surgery. Because of our patient's physical condition and his lifestyle-hampering symptomatology we chose was endovascular treatment in an attempt to recanalize the affected stent.

During the procedure it proved impossible after several attempts to recanalize the occluded stent in the SFA, probably because of already organized and fibrosed thrombus material in the stent. The crossover was not the cause of this failure, since the pushability and steerability of the guidewire-catheter assembly was sufficient. No angioplasty could be performed in the occluded stent. After several attempts we finally managed to pass the occluded stent subintimally, successfully re-entering the native lumen distally and creating a neo-lumen side-by-side with the primary occluded stent. The neo-lumen was stented to create enough radial force to prevent its elastic recoil. Two self-expandable stents were positioned in a double-barrel fashion alongside the previously occluded stent with proper clinical and angiographic result.

A retrograde puncture was preferred because of the lower complication rate compared with an antegrade approach in this obese male. Moreover a crossover introducer sheath assembly creates more possibilities for easier catheter-guidewire manipulations.

\section{Conclusion}

If a patient presents with claudication symptoms caused by in-stent restenosis or occlusion, one can try to recanalize the occluded stent. This is often not possible, especially if the occlusion is of an older date and consists of fibrosed tissue, mainly because of reorganizing thrombus. Mechanical debridement is an option, but 
this is more successful in (sub)acute (re)stenosis [3]. Subintimal passage of a occluded stent using the PIER technique could be an option in such cases. The PIER technique is intended as a primary therapy, usually for longer lesions of the SFA and for patients with severe (critical) limb ischemia, and has a 1-year patency rate of $22 \%$ and a success rate of $62 \%[1,2]$. As shown in our case, PIER appears to be good not only for recanalizing occlusions but also for providing options for the treatment of nonpenetrable in-stent occlusions by trespassing PIER-wise and subintimal stenting of the neo-lumen.

\section{References}

1. Yilmaz S, Sindel T, Yegin A, Lüleci E (2003) Subintimal angioplasty of long superficial femoral artery occlusions. J Vasc Interv Radiol 14:997_ 1010

2. Yilmaz S, Sindel T, Ceken K, Alimoglu E, Luleci E (2001) Subintimal recanalization of long superficial femoral artery occlusions through the retrograde popliteal approach. Cardiovasc Intervent Radiol 24:154-160

3. Berczi V, Deutschmann HA, Scheldbauer P, Tauss J, Hausegger KA (2002) Early experience and midterm follow-up results with a new, rotational thrombectomy catheter. Cardiovasc Intervent Radiol 25:275281 